\title{
Teamwork in the Hospital for the Patient
}

\author{
Extracts from papers read at the Symposium arranged by the S.A. Nursing Council, Johannesburg, in October
}

\section{Practical Suggestions for Better Team Work}

Each member of the team must be receptive and not resentful or suspicious or jealous of other team members. Each of us must continuously remind ourselves that we are all working for the patient.

\section{Physiotherapist}

When the physiotherapist reports redness of the skin and a tendency to break down, she is not criticising the nursing of that patient. We all know how quickly a pressure sore will develop in a paraplegic patient and how easily it can be prevented and how many unhappy wasted months in bed can result if it is not prevented. This and this only should be the reason why the physiotherapist approaches the nurse and why the nurse in turn co-operates - the welfare of a patient is at stake and the pride of individuals must take second place.

Co-operation on both sides is required as far as timing of treatments with applications of dressings goes. A good arrangement can be made that the nurse cleans the sores first thing in the morning and covers them with a sterile lotion cloth. Then the physiotherapist comes and applies her treatment, replaces the cloth until the nurse returns to redress the wound. Ultra-violet Radiation and Ultra-sound for sores works best in combination with good nursing, that is the patient is kept dry and clean and pressure free day and night.

Pressure sores should not exist at all and with proper knowledge, co-ordination and supervision they can be avoided.

Liaison between doctors and auxiliaries through the ward sister should be so efficient as to make up for not going on ward rounds when this is impossible. The physiotherapist should lodge queries via the sister, who in turn can convey all relevant information to her. The sister, co-ordinator of the team must therefore have a full understanding and the work of all the auxiliaries - a no mean feat.

Ward classes, so valuable to the bed patient provide another example of co-operation between nursing and physiotherapy staff, because of the inevitable interruption of ward routine.

Plans for discharge of a patient should be conveyed well in advance to all team members so that the patient can receive instructions about home exercises, out-patient treatment, etc. After perhaps months of toil with one patient it is so disheartening to find he is discharged with no prior warning or time for instruction.

\section{The Nurse}

Extracts from this paper will be published in the next issue, if possible.

\section{Radiographer}

The examination most frequently done in X-ray is investigation of the lungs. After chest surgery the radiographer must take immediate X-rays to assess re-expansion of the lungs and, to assist continuity of the patient's care, these $\mathrm{X}$-rays are then shown as soon as possible to the physiotherapist, who commences her treatment on these findings. It is necessary for the physiotherapist to know exact localities of lesions so that she may accurately drain the areas affected. It is therefore important that the X-ray results are conveyed to her immediately by the sister of the ward or through a note from the doctor on the bed letter.

When patients are sent in for X-ray of any sort it is important that the clothing and appliances worn should be able to be easily and quickly removed both for the success of the X-ray and the ease and comfort of the patient.

\section{Occupational Therapist}

Kinetic Occupational Therapy is the type of treatment usually given in the Occupational Therapy department and in this field physiotherapists and occupational therapists should give treatments which compliment each other. Where there is good liaison between these two members of the Paramedical staff patients receive most benefit from the treatments given.

Activities of Daily Living or A.D.L. often conveys no meaning to doctors, nurses and paramedical staff, and yet this is a very important part of occupational therapy. When a paraplegic patient becomes mobile and attends the A.D.L. Unit do not help him in the wards. The occupational therapist spends hours teaching the paraplegic to manage without assistance and yet so often if she walks into the ward she finds the patient sitting back while the nurse assists him to dress or put on his calipers. Nurses and occupational therapists can co-operate in such a way as to make the patient an independent person. This applies to the use of gadgets as well, designed in O.T. to assist patients in eating, dressing or any other activity of daily living. 